\title{
All-Optical Two-mode Switching in Semiconductor Ring Lasers
}

\author{
Alessandro Scirè ${ }^{*}$, Marc Sorel $^{b}$, Guido Giuliani ${ }^{c}$, \\ ${\text { Pere } \text { Colet }^{a} \text {, Toni Pérez }}^{a, d}$, Claudio R. Mirasso ${ }^{d}$ \\ ${ }^{a}$ Instituto Mediterráneo de Estudios Avanzados (IMEDEA), \\ Consejo Superior de Investigaciones Científicas-Universitat de les Illes Balears, \\ Campus Universitat Illes Balears, E-07122 Palma de Mallorca, Spain. \\ ${ }^{b}$ Department of Electronic and Electrical Engineering, \\ University of Glasgow, Rankine building, Oakfield Ave. \\ Glasgow G12 8LT, Scotland, UK. \\ ${ }^{c}$ Dipartimento di Elettronica, Università di Pavia, \\ Via Ferrata 1, I-27100 Pavia, Italy. \\ ${ }^{d}$ Departament de Física, Universitat de les Illes Balears. \\ E-07122 Palma de Mallorca, Spain.
}

\begin{abstract}
We have theoretically investigated the bifurcation scenario that leads to the emergence of a bistable regime in a twomode model for a Semiconductor Ring Laser. The bistability takes place between two quasi-unidirectional solutions for the electric field, which are selected as stable solutions via gain-crossaturation, for well-above threshold operating conditions. Furthermore, we analyzed the switching properties of a single Semiconductor Ring Laser (SRL) operating in the bistable regime, under coherent optical pulse injection, in view of the possible implementation of a single SRL an optically adressable memory element. The result is that the response time and the minimum switching energy respectively attain values the order of a few tenth of $p s$, and $1 f J$. Those values are espected to scale down with the device radius, due to the consequent decreasing of the cavity flight time. We have observed that the fast switching dynamic is due to an energy redistribution process between the two counterpropagating modes, that does not involve the (slow) carrier density through field-medium energy exchange processes. This allows to attain time scales much faster than the typical limit represented by the inverse of relaxation oscillation frequency.
\end{abstract}

\section{INTRODUCTION}

Ring lasers have been the subject of a huge amount of experimental and theoretical investigations, ${ }^{1}$ ranging from fundamental studies of its non-linear dynamics, ${ }^{2,3}$ to practical applications as the ring laser gyroscope. ${ }^{4}$ Recently, Semiconductor Ring Lasers ${ }^{5}$ (SRLs) have been investigated due to their peculiar two-mode dynamic properties. In particular, bistable operation has been demonstrated in several structures, namely single SRL, ${ }^{6-8}$ double SRL sharing the same active element, ${ }^{9}$ and passive silicon rings. ${ }^{10}$ Recently, the study of bistability in a tandem of two SRLs ${ }^{11}$ unveiled higly appealing features for applications in all-optical switching and optical memories. Indeed, the switching time of such structure has been experimentally proved to be fast and low consuming, enhancing the efforts toward a full understanding of the bistable SRL dynamics.

In this work, we theoretically analyze -in terms of its bifurcation scenario -the emergence of asymmetric solutions in a two-mode model for Semiconductor Ring Laser (SRL). We numerically investigate the switching properties of a single SRL operating in the bistable regime, under coherent optical pulse injection. We show that the fast switching dynamics is due to an energy redistribution process between the CW and CCW modes, that does not involve the (slow) carrier density variable.

* Author information: (Sent correspondence to A.S.): Email: scire@imedea.uib.es; Telephone: +34-971-171314; Fax: +34-971-173426; URL: http//www.imedea.uib.es

Semiconductor Lasers and Laser Dynamics II, edited by Daan Lenstra, Markus Pessa, lan H. White,

Proc. of SPIE Vol. 6184, 618408, (2006) · 0277-786X/06/\$15 · doi: 10.1117/12.661470 


\section{THE MODEL}

The theoretical analysis of the two-mode ring laser is based on a set of dimensionless semiclassical Lamb equations for the two (slowly varying) complex amplitudes of the counter-propagating fields $E_{1}\left(\mathrm{CW}\right.$ mode) and $E_{2}(\mathrm{CCW}$ mode), which has provided a good quantitative description of the two-mode dynamics in SRLs. ${ }^{6,7}$

The equations read:

$$
\dot{E}_{1,2}=(1+i \alpha)\left[N\left(1-s\left|E_{1,2}\right|^{2}-c\left|E_{2,1}\right|^{2}\right)-1\right] E_{1,2}-\left(k_{d}+i k_{c}\right) E_{2,1}+\frac{\tau_{p}}{\tau_{i n}} F_{1,2}(t)+\sqrt{\beta \tau_{p} N} \zeta_{1,2}(t),
$$

where $\alpha$ accounts for phase-amplitude coupling, the self and cross saturation coefficients are given by $s$ and $c$ respectively; the parameters $k_{d}$ and $k_{c}$ represent the dissipative and conservative components of the backscattered field, respectively. The term $F_{1,2}(t)$ represents resonant (zero-detuning) optical injection in the two modes, and it will be used to trigger the switching. Formally, this term is introduced according to the standard theory of injection in semiconductor laser, ${ }^{12,13} \tau_{p}$ is the photon lifetime, and $\tau_{i n}$ the flight time in the ring cavity. The last term of Eq.(1) represents spontaneous emission noise through Langevin forces, $\beta$ represents the fraction of the spontaneously emitted photons coupled to mode 1 or 2 , and $\zeta_{1,2}$ are two independent complex Gaussian random numbers, with zero mean $\left\langle\zeta_{i}(t)\right\rangle=0$ and correlation $\left\langle\zeta_{i}(t) \zeta_{j}^{*}\left(t^{\prime}\right)\right\rangle=2 \delta_{i j} \delta\left(t-t^{\prime}\right)$. The carrier density $N$ obeys the usual rate equation for semiconductor lasers,

$$
\dot{N}=\gamma\left[\mu-N-N\left(1-s\left|E_{1}\right|^{2}-c\left|E_{2}\right|^{2}\right)\left|E_{1}\right|^{2}-N\left(1-s\left|E_{2}\right|^{2}-c\left|E_{1}\right|^{2}\right)\left|E_{2}\right|^{2}\right],
$$

where $\mu$ is the dimensionless pump ( $\mu=1$ at laser threshold). In the set (1)-(2) the dimensionless time is rescaled by the photon lifetime $\tau_{p}$. The parameter $\gamma$ is the ratio of $\tau_{p}$ over the carrier lifetime $\tau_{s}$.

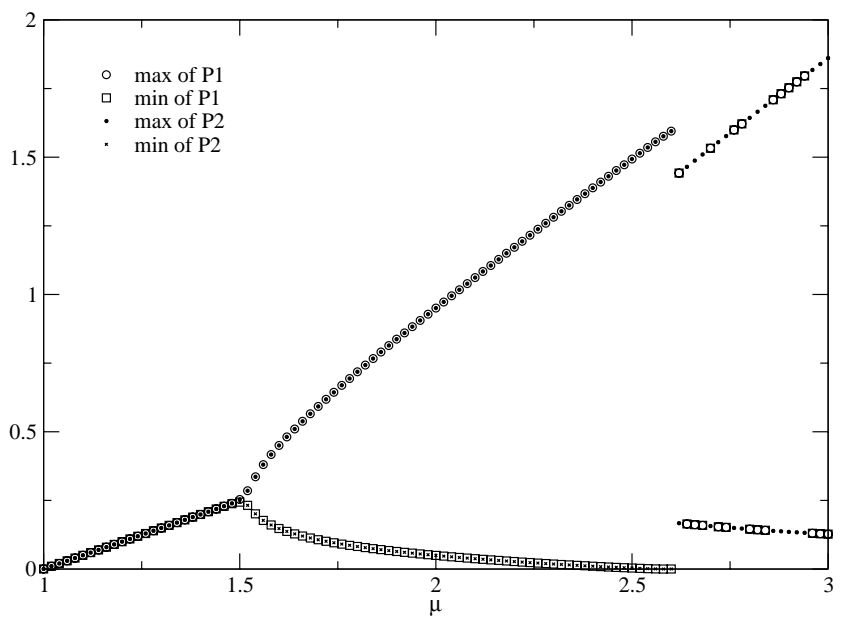

Figure 1. Bifurcation diagram for the fields intensities $P_{1,2}=Q_{1,2}^{2}$.

\section{ASYMMETRIC SOLUTIONS AND SWITCHING CHARACTERISTICS}

The set (1)-(2) ( with the following parameters choice $\alpha=3.5, s=0.005, c=0.01, k_{c}=0.0044, k_{d}=0.00327$, $\gamma=0.002$, and $\tau_{p}=10 \mathrm{ps}, \mu=3, \tau_{i n}=0.6 \mathrm{ps}$ ) shows the following bifurcation scenario (see Fig.1). The solutions of the set (1)-(2) can be expressed in the form $E_{1,2}=Q_{1,2} \exp \left(i \omega t+i \phi_{1,2}\right)$, and the corresponding value for $N$. At $\mu=1$ laser oscillation takes place. The presence of dissipative backscattering $\left(k_{d}\right)$ favors the presence of two symmetric solutions $\left(Q_{1}=Q_{2}\right)$ just above threshold, despite the presence of strong cross-saturation between the two modes. The two symmetric solutions, have the same field amplitude and carrier inversion but the relative phase $\left(\Phi=\phi_{2}-\phi_{1}\right)$ 
are $\Phi=0$ for the In-Phase Symmetric Solution (IPSS) and $\Phi=\pi$ the Out-of-Phase Symmetric Solution (OPSS). For negative (positive) values of $k_{d}$ the IPSS (OPSS) is stable. At $\mu \sim 1.5$ a Hopf bifurcation leads to the appearence of a limit cycle in the plane $\left(P_{2}-P_{1}, \Phi\right)$, which leads to alternate oscillations for the (experimentally accessible ${ }^{6,7}$ counter-propagating field intensities $\left(Q_{1,2}\right.$, respectively) at constant total intensity $\left(Q_{1}^{2}+Q_{2}^{2}\right)$ and carrier inversion $N$. Those oscillations are driven by the conservative part of the back-scattering coefficient $k_{c}$, and represents a dynamic competition between the two counterpropagating modes, which ends - for increasing values of $\mu$ - with the emergence of two stable asymmetric solutions, in which the optical power is mainly concentrated in one propagation direction. Physically, for high pump values, the strong cross-saturation between the two counterpropagting waves tends to favours unidirectional behavior. In this regime the device shows bistability between the two asymmetric solutions, and we call it bistable regime. Figure 2 shows some representative examples of the three kinds of solutions shown by the set (1)-(2).

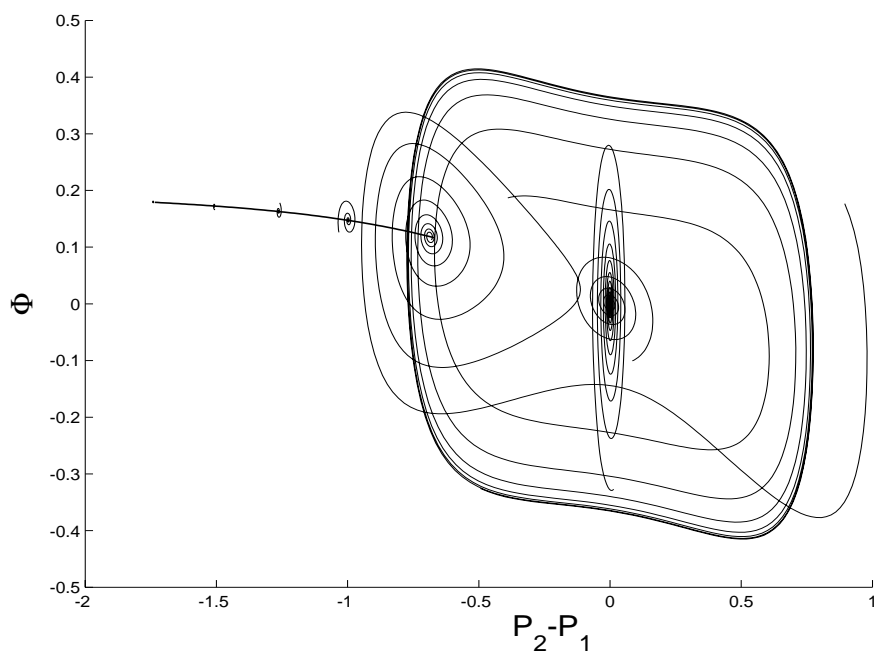

Figure 2. Examples of trajectories leading to the three different solutions, symmetyric solution $(\mu=1.6$, and $\mu=1.8)$, limit cycle $(\mu=2.0)$, and asymmetric solutions $(\mu=2.2, \mu=2.4, \mu=2.6$, and $\mu=2.8)$. The thick line represents the asymmetric solutions numerically calculated from the set (1)-(2). $\Phi=\phi_{2}-\phi_{1}, P_{1,2}=Q_{1,2}^{2}$.

In this section we focus on the bistable regime. The two stable states consist in laser emission mainly concentrated in one propagation direction - i.e. quasi ClockWise, qCW or quasiCounterClockwise (qCCW) - with a contrast factor $C=\frac{\left|E_{1}\right|^{2}-\left|E_{2}\right|^{2}}{\left|E_{1}\right|^{2}+\left|E_{2}\right|^{2}}$ that increases with $\mu$ from the Hopf bifurcation point. If no injection is applied, the system relaxes to one of the two stable states. If a pulse is applied in the opposite propagation direction (e.g. CCW), a switching occurs if the pulse energy exceeds a critical amount. We chose an optical trigger as a spike pulse of the form $F_{1,2}=A \exp (-t / \tau)$, characterized by the pulse amplitude $A$, and the pulse decay time $\tau$. The trigger amplitude $A$ is in general complex, due to the (constant) dephasing accumulated by the external field in the optical path outside the laser cavity. However, it is known that such associated phase does not affect the injection properties, ${ }^{13}$ and consequently we assume $A$ to be real.

We numerically simulated Eqs (1)-(2) through a standard fourth order Runge-Kutta algorithm. The optical injection was a stream of 1000 trigger pulses (alternating the $\mathrm{CW}$ and $\mathrm{CCW}$ ) at a constant rate of $1 \mathrm{GHz}$, to generate the statistics. Numerical simulation (see Fig.3) show that if the system is in the $\mathrm{qCW}(\mathrm{qCCW})$ state and a $\mathrm{CCW}(\mathrm{CW})$ pulse is applied, a switching occurs to the $\mathrm{qCCW}(\mathrm{qCW})$ state if the switching energy exceeds a critical amount. We computed the normalized cross-correlation between $P_{1}=\left|E_{1}\right|^{2}$ and $P_{2}=\left|E_{2}\right|^{2}$, evaluated at a time delay equal to the trigger period $T=(1 G H z)^{-1}=1 \mathrm{~ns}$, that is

$$
X(T)=\frac{\frac{1}{T_{\max }} \int_{0}^{T_{\max }} P_{1}(t) P_{2}(t-T) d t}{\bar{P}_{1} \bar{P}_{2}},
$$




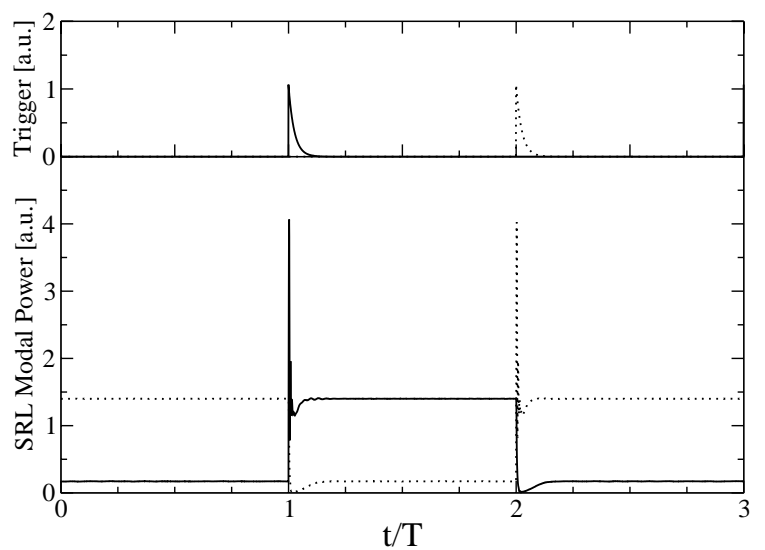

Figure 3. Time traces of the dynamic variables under pulsed optical injection operation conditions. Upper panel: dimensionless trigger amplitude $\left|F_{1}(t)\right|$ (thick), and $\left|F_{2}(t)\right|$ (dashed). Lower panel: dimensionless modal amplitudes $\left|E_{1}(t)\right|$ (thick), and $\left|E_{2}(t)\right|$ (dashed). The trigger characteristics: $A=0.1, \tau=0.1 T, T=1$ ns.

where $T_{\max }=1000 \mathrm{~T}$ is the total integration time, $\bar{P}_{1} \sim \mu-1 \mathrm{~s}$ the average values of the intensity $\left|E_{1}\right|^{2}$ when the (solitary) qCW mode is active (the same holds for the CCW mode intensity $\bar{P}_{2}$ ). Note that the normalization procedure we used allows $X(T)$ to be slightly larger than one, due to the power injected by the trigger. If all the trigger pulses induce a switching $X(T) \sim 1$, whereas if some switching events fail $X(T)$ decreases and approaches to zero if no switching occurs. We have computed the value of $X(T)$ for different values of the trigger amplitude and decay time, and the result is shown in Fig.4. Numerical simulations showed that the shape of the pulse is not critical for the switching to occour, being the relevant magnitute its energy, given by $\varepsilon=\int_{0}^{\infty}\left|F_{1,2}\right|^{2} d t=A^{2} \tau / 2$.

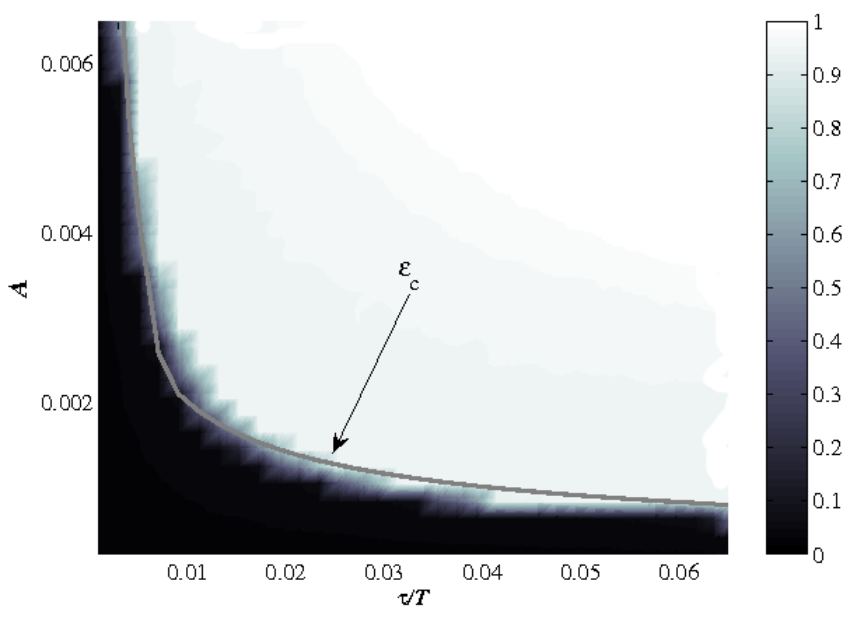

Figure 4. Cross-correlation function $X(T)$, given by Eq.(3), versus the dimensionless trigger pulse amplitude $A$, and the pulse decay time $\tau$ normalized to the pulse repetition rate $\mathrm{T}=1 \mathrm{~ns}$. Parameters as in Fig3. The figure shows that the transition occours in corrispondence of a iso-energy curve for the trigger pulse, individuating a minimum (critical) switching energy $\varepsilon_{c} \sim 1 f J$,which has been calculated assuming $100 \mathrm{~mW}$ of optical power inside the cavity, in agreement with the measured output optical power and coupler efficiency in real devices. 
Indeed,Fig.4 shows a transition to fully successful switching events if the trigger pulse energy exceeds a critical value $\varepsilon_{c} \sim 1 f J$. With the same data, and after each pulse is applied, we calculate the time it takes the system to reach one half of the value of the intensity of the final state $\left(\bar{P}_{1,2}\right)$. This time $\left(t_{R}\right.$ from now on) is an estimation of the response time of the system to the trigger pulse, and characterizes the switching speed. Due to the presence of noise, $t_{R}$ underogoes a statistic distribution, that we characterize through its mean value $\left\langle t_{R}\right\rangle$ and variance $\sigma_{R}=\sqrt{\left.<t_{R}^{2}>-<t_{R}\right\rangle^{2}}$ for different values of the trigger amplitude, as it is shown in Fig.5. Fig.5 shows that a fast response time (about $30 \mathrm{ps}$ ) can be attained.

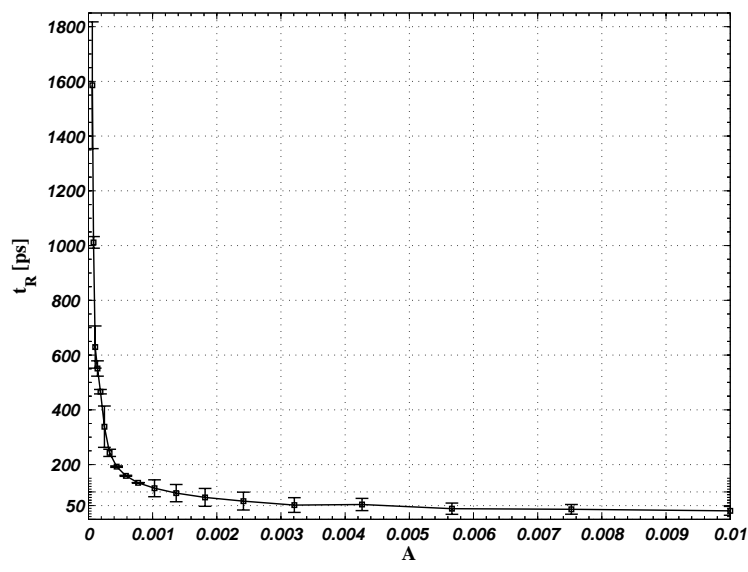

Figure 5. Statistics of the response time $t_{R}$ Vs dimensionelss trigger pulse amplitude $A$. dots: mean value $<t_{R}>$; error bars $\sigma_{R}=\sqrt{<t_{R}^{2}>-<t_{R}>^{2}} \cdot \tau / T=0.1$

The obtained response time is throughout much lower than the inverse of the relaxation oscillation frequency $\left(\tau_{\text {rel.ox }}=f_{\text {rel.ox }}^{-1}\right.$, where $\left.f_{\text {rel.ox }}=\frac{1}{2 \pi \tau_{p}} \sqrt{\gamma(\mu-1)} \sim 1 G H z\right)$ for our parameter values. By analyzing the time evolution of the dynamical variables of Eqs (1)-(2), we observed that the the carrier density $N$ remain almost unaffected during the switching process (see. Fig. 5). Physically, this accounts for the fact that the switching itself represents a energy redistribution between the two states of the electric field. Thus no field-medium energy exchange processes come significantly into play during the switching. This fact permits the switching itself to attain time scales much faster than the one that limits the field-medium energy exchange process: the inverse of the relaxation oscillation frequency.

\section{CONCLUSIONS}

In conclusion, we have theoretically investigated the emergence of a bistable regime in a two-mode model for a Semiconductor Ring Laser. The bistability takes place between two quasi-unidirectional solutions for the electric field, which are selected as stable solutions via gain-crossaturation, for well-above threshold operating conditions. Furthermore, we analyzed the switching properties of a single Semiconductor Ring Laser (SRL) operating in the bistable regime, under coherent optical pulse injection, in view of the possible implementation of a single SRL an optically adressable memory element. The result is that the response time and the minimum switching energy respectively attain values the order of a few tenth of $p s$, and $1 f J$. Those values are espected to scale down with the device radius, due to the consequent decreasing of the cavity flight time. In principle, faster ( $p s$ or sub-ps) time scales would require more sophisticated (e.g. travelling-wave) modeling approach, and will be the subject of future investigations. However, we have observed that the fast switching dynamic is due to an energy redistribution process between the two counterpropagating modes, that does not involve the (slow) carrier density through field-medium energy exchange processes. This allows to attain time scales much faster than the typical limit represented by the inverse of relaxation oscillation frequency. 


\section{Acknowledgements}

This work has been funded by the Spanish MCyT and Feder under project TEC2005-07799-C02-01 and FIS200400953. A.S. acknowledges Marie Curie IF MCFI-2000-00617, and Ramon y Cajal program by MCyT. The authors acknowledge Raúl Vicente for useful discussions.

\section{REFERENCES}

1. C. O. Weiss and R. Vilaseca, Dynamics of lasers, Weinheim, New York (1991), and refs therein.

2. Q. L. Williams, and R. Roy, Optics Letters, 37, 470 (1988).

3. H. Nakatsuka, S. Asaka, H. Itoh, K. Ikeda, and M. Matsuoka, Phys. Rev. Lett, 50, 109 (1983); E. J. D’Angelo, E. Izaguirre, G. B. Mindlin, L. Gil, and J. R. Tredicce, Phys. Rev. Lett, 68, 3702 (1992).

4. W. W. Chow, J. Gea-Banacloche, L. M. Pedrotti, V. E. Sanders, W. Schleich and M. O. Scully, Rev. of Mod. Phys. 5761 (1985).

5. T. Krauss, P. J. R. Laybourn and J. S. Roberts, Electron. Lett. 26, 2095 (1990).

6. M. Sorel, J. P. R. Laybourn, A. Scirè, S. Balle, G. Giuliani, R. Miglierina, S. Donati, Optics Letters 27, 1992 (2002).

7. M. Sorel, G. Giuliani,A. Scirè, R. Miglierina, J. P. R. Laybourn, S. Donati, IEEE J. Quantum Electron. 39, $1187(2003)$.

8. J. J. Liang, S. T. Lau, M. H. Leary and J. M. Ballantyne, Applied Phys. Lett. 70, 1192 (1997).

9. S.Zhang, Y. Liu, D.Lenstra, M.T.Hill, H.Ju, G.D. Khoe, H.J.S.Dorre, J. Selected Topics Q. Electronics 10, 1093 (2004).

10. V. R. Almeida, C. A. Barrios, R. P. Panepucci, M. Lipson, M. A. Foster, D. G. Ouzounov, A. L. Gaeta, Optics Letters, 29, 2867 (2004).

11. M. T. Hill, H. J. S. Dorren, T. de Vries, X. J. M. Leijtens, J. H. den Besten, B. Smalbrugge, Y. S Oei, H. Binsma, G. D. Khoe, M. K. Smit, Nature 432, 206 (2004).

12. G. H. M. van Tartwijk, D. Lenstra, Quantum Semiclass. Opt. 7, 87 (1995).

13. V. Annovazzi-Lodi, A. Scirè, M. Sorel,S. Donati, IEEE J. Quantum Electron. 34, 2350 (1998). 